\title{
Breast cancer screening pamphlets mislead women
}

All women and women's organisations should tear up the pink ribbons and campaign for honest information

\section{Gerd Gigerenzer director}

Harding Center for Risk Literacy and Centre for Adaptive Behaviour and Cognition, Max Planck Institute for Human Development, Berlin, Germany

Why should I have mammography? That question is regularly asked in pamphlets for screening. The answer is also regularly misleading. Women are told what they should do, but without being given the facts necessary to make informed decisions. This form of paternalism has a long tradition. In a campaign poster in the 1980s, the American Cancer Society declared: "If you haven't had a mammogram, you need more than your breasts examined."

As a result of paternalism and pink ribbon culture, almost all women have a false impression of the benefit of mammography screening. For instance, 98\% of women in France, Germany, and the Netherlands overestimated its benefit by a factor of 10 , 100 , or more, or did not know. ${ }^{1}$ Most surprisingly, those who frequently consulted their physicians and health pamphlets were slightly worse informed. Russian women gave the most realistic estimates among those in nine European countries studied-not because they have more information at their disposal but because there are fewer misleading pink ribbon pamphlets in Russia.

Misinformation needs to stop. All pamphlets should show a "fact box" that explains benefits and harms in a transparent way. ${ }^{2}$ The figure shows one based on the most recent Cochrane review for women age 50 to $69 .^{3}$

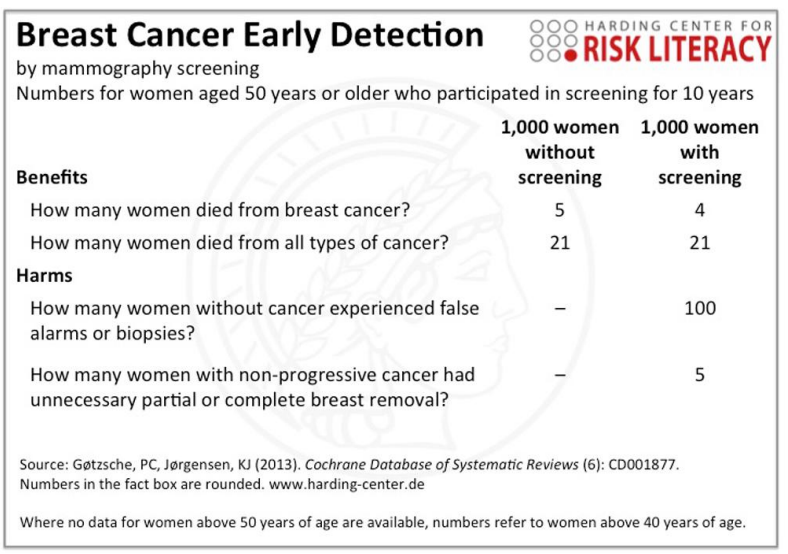

In sum, the absolute reduction in mortality from breast cancer is about 1 in 1000 women, but the reduction in total cancer mortality (including breast cancer) is 0 . The difference between breast cancer and total cancer deaths is important because it is not always easy to determine the type of cancer from which a person died, and total cancer mortality is thus a more reliable measure.

A look at a sample of pamphlets reveals patterns in how the benefits of screening are actually communicated (for the sake of brevity, I do not deal with the harms). Four strategies are frequently used:

\section{Zero number policy: tell women what to do without stating benefits}

Even today, woman are simply told to go for mammographic screening and are given no detailed estimates of the benefit. In the US the Food and Drug Administration's Office of Women's Health leaflet (in pink) says on its first page that "Mammograms can help save lives." Similarly, the American Cancer Society's 2014 pamphlet Breast Cancer: Early Detection tells women, "Most doctors feel that early detection tests for breast cancer save thousands of lives each year, and that many more lives could be saved if even more women and their health care providers took advantage of these tests," and the National Cancer Institute's fact sheet says, "Screening mammography can help reduce the number of deaths from breast cancer among women ages 40 to 70 , especially for those over age 50."

In each case, no information is given about how large the benefit is. In the first two cases, the reduction in breast cancer mortality is misleadingly presented as "saving lives," even though there is no reduction in total cancer mortality (including breast cancer): no life is actually saved. Note the American Cancer Society's formulation that most US doctors "feel" that lives are saved, which may be technically true. This zero number policy seems to be widespread in the US, unlike in Canada and the rest of the Western world. 


\section{Report relative risks only}

The second strategy is to report the reduction in breast cancer mortality as a relative risk rather than absolute risk reduction. That is, the reduction from $5 / 1000$ to $4 / 1000$ is expressed as a $20 \%$ reduction, sometimes generously rounded up to over $30 \%$. This makes the benefit look larger than the $0.1 \%$ absolute reduction. The Welsh NHS leaflet Breast Screening Explained says, "Breast screening has been shown to reduce the risk of dying from breast cancer by around 35\%." And one by the New Zealand Breast Cancer Foundation claims that "Screening mammograms ... reduce the chance of dying from breast cancer by approximately $33 \%$."

None of these pamphlets tells women that there is no difference in total cancer mortality.

\section{Report five year survival rates}

The third strategy is to use another misleading statistic: five year survival rates. It is well known that these rates say nothing about mortality reduction. In fact, increases in survival rates are not even correlated with decreases in mortality rates, $r=0.0{ }^{4}$

Lead time bias (diagnosis of breast cancer through screening at an early stage that does nothing but advance the date of diagnosis) and overdiagnosis (diagnosis of a type of breast cancer that would never cause symptoms or death during a woman's lifetime) inflate five year survival rates without reducing mortality. ${ }^{4}$ Nevertheless, high survival rates continue to be used to impress women. For example, the Avon

Foundation's breast health resource guide says, "There is a $97 \%$ 5 -year survival rate when breast cancer is caught early before it spreads to other parts of the body."

\section{Report absolute risk reduction but use unrealistically high numbers}

Several pamphlets have stopped reporting misleading relative risks and five year survival rates. They report understandable absolute risks but inflate these. The leaflet produced by BreastScreen Australia states: "For every 1000 women who are screened every two years from age 50 to age 74 through BreastScreen (over 25 years): around 8 (between 6 and 10) deaths from breast cancer will be prevented." And the NHS leaflet for England tells women, "Screening saves about 1 life from breast cancer for every 200 women who are screened."
One way to artificially inflate the absolute risk reduction (for about 10 years, as reported in the fact box) is to assume that the benefit will increase linearly if you consider 25 years (as BreastScreen does). But there is no evidence for this assumption. The only study that has actually investigated risk over 25 years found no reduction of breast cancer deaths at all. ${ }^{5}$

\section{A right to be informed}

In Germany, the Harding Center for Risk Literacy (of which I am a director) successfully exposed health organisations for misinforming the public about mammography screening. As a consequence, since about 2010, all deceptive relative risks and five year survival rates have been removed from German information literature, and harms are now reported in absolute numbers. Thus far, however, no German organisation has dared to publish a fact box. In Austria, the Tyrolean Society for General Medicine did and was immediately attacked by representatives of the local gynaecology departments. The leaflet of the Canadian Task Force Should I be screened with mammography for breast cancer? is another good example of how to inform women honestly.

I call on all women and women's organisations to tear up the pink ribbons and campaign for honest information. Only by correcting the current misinformation rate of $98 \%$ in various countries will women be in a position to make informed decisions.

Competing interests: I have read and understood BMJ policy on declaration of interests and have no relevant interests to declare.

Provenance and peer review: Commissioned; not externally peer reviewed.

Gigerenzer G, Mata J, Frank R. Public knowledge of benefits of breast and prostate cance screening in Europe. J Natl Cancer Inst 2009;101:1216-20.

2 Schwartz LM, Woloshin S. Using a drug facts box to communicate drug benefits and harms: two randomized trials. Ann Intern Med 2009;150:516-27.

3 Gøtzsche PC, Jørgensen KJ. Screening for breast cancer with mammography. Cochrane Database Syst Rev 2013;6:CD001877.

4 Welch HG, Schwartz LM, Woloshin S. Are increasing 5-year survival rates evidence of success against cancer? JAMA 2000:283:2975-8.

5 Miller AB, Wall C, Baines CJ, Sun P, To T, Narod SA. Twenty five year follow-up for breast cancer incidence and mortality of the Canadian National Breast Screening Study: randomized screening trial. BMJ 2014;348:g366.

Cite this as: BMJ 2014;348:g2636

๑ BMJ Publishing Group Ltd 2014 